Orinoquia, Enero-Junio 2020;24(1): 13-22

ISSN electrónico 2011-2629.

ISSN impreso 0121-3709.

http://doi.org/10.22579/20112629.587

\title{
Captura de carbono en biomasa de sistemas de uso del suelo, municipio de Yopal, Casanare, Colombia
}

\author{
Carbon capture regarding biomass from rural land use systems near \\ the municipality of Yopal, Casanare, Colombia
}

\section{Captura de carbono na biomassa de sistemas de uso da terra, município de Yopal, Casanare, Colômbia}

Blanca N. Carvajal-Agudelo ${ }^{1}$, Hernán J. Andrade ${ }^{2}$

1 Ing. Forestal, Esp, MSc, Estudiante de Maestría en producción tropical sostenible, Facultad de Ciencias Agropecuarias y Recursos Naturales, Universidad de los Llanos, Villavicencio-Meta, Colombia.

2 Ing. Agron, MSc, PhD, Grupo de Investigación PROECUT, Facultad de Ingeniería Agronómica, Universidad del Tolima. Ibagué, Tolima, Colombia.

Email: forestalninfa@hotmail.com

Recibido: 14 de marzo de 2019

Aceptado: 11 de marzo de 2020

\begin{abstract}
Resumen
El cambio climático, ocasionado por el incremento en la concentración de gases efecto invernadero (GEl), genera alteraciones en el clima del planeta, aumentando la temperatura media global, lo que afecta patrones de precipitación. El área de estudio se ubicó en el Municipio de Yopal, corregimiento Tacarimena, compuesta por ocho veredas, la cual presenta clima cálido - húmedo con promedio de precipitación anual de $2270 \mathrm{~mm}$; temporada seca de diciembre-marzo y lluviosa de abril-noviembre y alturas inferiores a $380 \mathrm{~m}$. En concordancia con la necesidad del desarrollo bajo en carbono, la presente investigación estima la biomasa arriba y abajo del suelo y con éstas el carbono total almacenado en siete sistemas de uso del suelo: 1) plátano con sombrío (SAF+ plátano), 2) cacao con sombrío (Ca+S), 3) cítricos (C), 4) sistema silvopastoril bajo (SSPB), 5) sistema silvopastoril alto (SSPA), 6) bosques de galería (BG), y 7) mata de monte (MM). Se trabajó con diseño experimental completamente al azar con cinco repeticiones, para un total de 35 unidades experimentales. Se establecieron parcelas temporales de muestreo, tomando datos en 832 árboles de 66 especies botánicas. Se estimó la biomasa arriba del suelo mediante modelos alometricos, utilizando datos de campo (diámetro a la altura del pecho dap y la altura total). La biomasa abajo del suelo (raíces) se estimó empleando el modelo general para bosques tropicales. Todos los usos del suelo en estudio ofrecen el servicio ecosistémico de captura de carbono, siendo el BG y la MM los de mayor carbono, mientras que el SAF+plátano almacenó la menor cantidad de carbono. Potenciales cambios de sistemas productivos a sistemas forestales (BG y MM) implican una ganancia de carbono (adicionalidad), mientras que los cambios contrarios, es decir deforestación, representan emisiones de $\mathrm{CO}_{2}$. Estos resultados son claves para la orientación a políticas y proyectos de captura de carbono.
\end{abstract}

Palabras clave: Biomasa; cambio climático; ecuación alométrica; emisión; mitigación; sistema agroforestal 


\begin{abstract}
Climate change caused by increased greenhouse gas (GHG) concentration causes alterations in the planet's climate and increases the average global temperature, thereby affecting rainfall patterns. This study's target area was the town of Tacarimena in the municipality of Yopal; it has eight rural areas. The area is located around 380 masl and has a warm, humid climate, a mean annual rainfall of 2,270 mm, a dry season between December and March and a rainy season from April to November. This research has estimated seven landuse systems' above- and below-ground biomass and total carbon storage in line with a low-carbon development policy: 1) plantain with shade (SAF + plantain), 2) cocoa with shade $(\mathrm{Ca}+\mathrm{S}), 3)$ citrus $(\mathrm{C}), 4)$ low-lying silvopastoral system (LSS), 5) high-lying silvopastoral system (HSS), 6) gallery forest (GF) and 7) bush (B). A completely randomised experimental design with five repetitions was used, giving 35 experimental units. Temporary sampling plots were established for taking information regarding 832 trees from 66 botanical species. Allometric models were used for estimating above-ground biomass using field data/measurements (diameter at breast height (DBH) and total height (TH). A general tropical forest model was used for estimating below-ground biomass. All the land-use systems being studied had the essential ecosystem service of carbon capture/CO2 sequestration where GF and B had the highest carbon storage; on the contrary, SAF + plantain stored the lowest amount of carbon. Changing from production to forestry systems (GF and B) implies increased carbon capture (additionality), whereas the opposite (i.e. deforestation) represents $\mathrm{CO} 2$ emission. Such results represent a key input for policy design and carbon capture projects.
\end{abstract}

Key words: Biomass; climate change; allometric equation; emission; mitigation; ecosystem service; agroforestry system.

\title{
Resumo
}

A mudança climática, causada pelo aumento da concentração de gases de efeito estufa (GEEs), gera alterações no clima do planeta e um aumento na temperatura média global, afetando os padrões de precipitação. A área de estudo foi localizada no município de Yopal, distrito Tacarimena, composto por oito vilarejos, que tem um clima quente - úmido com precipitação anual média de $2.270 \mathrm{~mm}$, além de uma estação seca de dezembro a março e uma estação chuvosa entre abril e novembro. De acordo com a necessidade de desenvolvimento baixo em carbono, esta pesquisa estima a biomassa acima e abaixo do solo e com eles o total de carbono armazenado em sete sistemas de uso da terra: 1) banana com sombra (SAF + banana), 2) cacau sombreado (Ca + S), 3) cítricos (C), 4) sistema silvopastoril baixo (SSPB), 5) sistema silvopastoril alto (SSPA), 6) floresta riparia (BG), e 7) fragmento de floresta (MM). Desenvolveu-se um desenho experimental inteiramente casualizado com cinco repetições, totalizando 35 unidades experimentais. Parcelas temporárias de amostragem foram estabelecidas, tomando dados de 832 árvores de 66 espécies botânicas. A biomassa acima do solo foi estimada com modelos alométricos, inserindo os dados de campo: diâmetro à altura do peito (dap) e a altura total. A biomassa abaixo do solo foi estimada usando um modelo geral para florestas tropicais. Todos os usos da terra em estudo oferecem o serviço ecossistêmico de captura de carbono, sendo BG e MM os que apresentam o maior carbono, enquanto que SAF + banana armazenou a menor quantidade de carbono. Mudanças potenciais de sistemas de produção para sistemas florestais (BG e MM) implicam em ganho de carbono (adicionalidade), entanto que as mudanças opostas, como o desmatamento, representam emissões de CO2. Esses resultados são chaves para orientar políticas e projetos de captura de carbono.

Palavras-chave: Biomassa; mudança climática; equação alométrica; emissão; mitigação; sistema agroflorestal

\section{Introducción}

Los cambios de uso del suelo son factores importantes para el balance global de carbono y representan una opción para el desarrollo de proyectos de mitigación del cambio climático (FAO, 2002; Alvarado et al., 2013). El $\mathrm{CO}_{2}$ presente en la atmósfera se captura por las plantas mediante los procesos metabólicos que corresponden al balance fotosíntesis-respiración. La captura de $C$ se expresa en términos de la biomasa constituida por el follaje, ramas, raíces, troncos, flores y frutos. Un incremento en la biomasa de un sistema de uso del suelo constituye una captura de carbono, lo cual implica la reducción de emisiones de gases de efecto invernadero (GEI) (IPCC, 2013).

El estado colombiano ha venido actuando en dirección a la gestión eficiente y el cumplimiento de los compromisos de la Conferencia de las Partes (COP 21) de la Convención Marco de las Naciones Unidas so- bre el Cambio Climático (CMNUCC, 2015). Colombia ha venido formulando e implementará políticas que permitan la protección y adaptación de su población a los efectos adversos ocasionados por el cambio climático mediante la implementación de acciones de mitigación de emisiones de GEI. En este sentido, El Congreso de Colombia (2018) promulgó la Ley 1931 de 2018, la cual establece "las directrices para la gestión del cambio climático lo que permite la toma de decisiones con el objetivo de reducir la vulnerabilidad de la población y de los ecosistemas del país frente a los efectos del mismo y promover la transición hacia una economía competitiva, sustentable y un desarrollo bajo en carbono". A pesar de la importancia de este tema, existen limitadas experiencias de investigación en captura y flujos de carbono en sistemas de uso del suelo de la Orinoquía colombiana.

El presente estudio plantea la hipótesis que "cambios en los usos del suelo causan alteraciones en el alma- 
cenamiento de carbono en biomasa arriba y abajo del suelo, lo cual puede causar emisiones de GEl o captura adicional de carbono".

Detectar los sistemas que permitan una mayor acumulación de carbono y aquellas tecnologías "ganarganar", por generar este servicio ambiental y a la vez obtener productos, es clave para el desarrollo de políticas y proyectos de carbono y/o conservación (Mena et al., 2011). El objetivo del estudio es estimar la captura de carbono en biomasa total y la potencial fijación de carbono o emisión de $\mathrm{CO}_{2}$ por cambios de uso del suelo; en el valle aluvial del río Cravo Sur, Yopal, Casanare, Colombia. En este sentido el estudio busca identificar los sistemas de uso del suelo que van en línea de la Estrategia Colombiana de Desarrollo Bajo en emisiones de Carbono (ECDBC), la cual identificó acciones de mitigación sectoriales para reducir las emisiones de GEl y, con este mismo esfuerzo, generar desarrollo económico, social y ambiental en el sector rural (MADS, 2011).

\section{Materiales y métodos}

\section{Localización del área de estudio}

El área de estudio se ubicó en el corregimiento de Tacarimena, que está compuesto por ocho veredas: El Nocuito, Manantiales; Sirivana, Palomas, La calceta, La Manga, Tacarimena y El Tiestal (Figura 1). La Alcaldía de Yopal (2013) indica que El "corregimiento de Tacarimena tiene una superficie de 33.925 ha, se localiza al suroriente del casco urbano del Municipio de Yopal, Casanare Colombia entre las coordenadas $5^{\circ} 22^{\prime} 09.8^{\prime \prime} \mathrm{N}-72^{\circ} 12^{\prime} 12.3^{\prime \prime} \mathrm{O}$; $5^{\circ} 17^{\prime} 23.58^{\prime \prime} \mathrm{N}-72^{\circ} 20^{\prime} 13.29^{\prime \prime} \mathrm{O} ; 5^{\circ} 11^{\prime} 52.88^{\prime \prime} \mathrm{N}-72^{\circ} 3^{\prime}$ $48.83^{\prime \prime O}$ y $5^{\circ} 11^{\prime} 29.56^{\prime \prime} \mathrm{N}, 72^{\circ} 10^{\prime} 57.32^{\prime \prime} \mathrm{O}$; haciendo parte de la cuenca media del Río Cravo Sur. Su paisaje es valle aluvial en vegas y terrazas, con suelos fértiles poco profundos sobre cantos rodados de lo que se denomina el Abanico de Yopal, de clima cálido húmedo".

CORPORINOQUIA (2019) afirma que los "suelos son ligeramente ácidos, con moderados contenidos de materia orgánica que varía irregularmente con la profundidad, presentan capacidad de cambio catiónico baja, saturación de bases moderada a alta y fertilidad natural, limitados también por contenidos tóxicos de aluminio". El IGAC (2014) indica que el "área de interés está conformada por suelos de la Asociación Fluventic Humic Dystrudepts-Typic Fluvaquents. Estos suelos se localizan anexo a ríos y quebradas del valle, en relieves planos, con pendientes menores del 3\%, área susceptible a las inundaciones que pertenece a la zona de vida Bosque Húmedo Tropical (bh-T)".

\section{Metodología experimental y mediciones en campo}

Se empleó un diseño completamente al azar con los usos del suelo como tratamientos:

1) Plátano con sombrío (SAF+plátano). En el sistema productivo en estudio las especies arbóreas asocia-

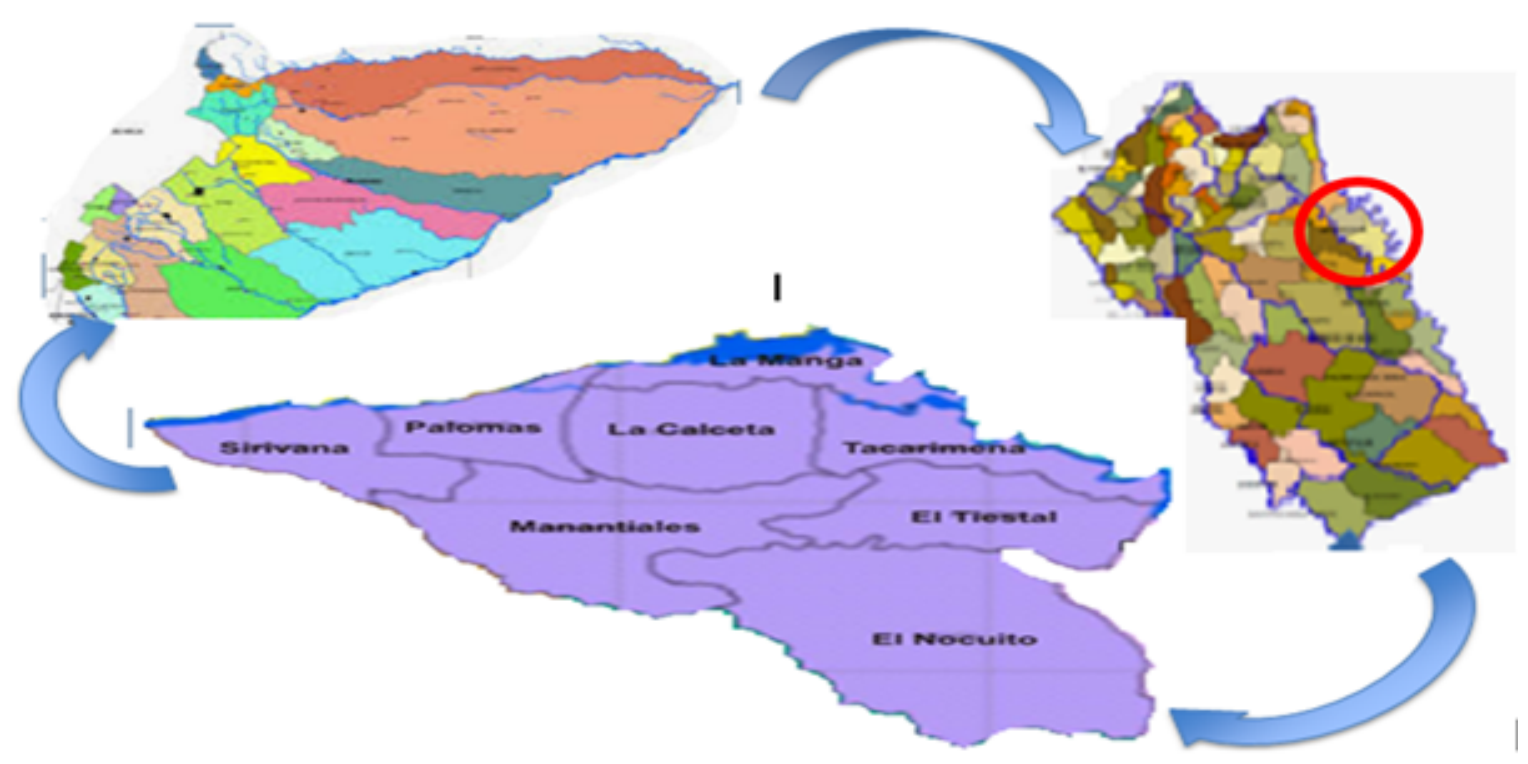

Fuente: Plan Básico de Ordenamiento territorial, 2013.

Figura 1. Localización del área de estudio en Corregimiento de Tacarimena, Municipio Yopal, Casanare, Colombia. 
das al cultivo son las siguientes: acacia mangium (Acacia mangium), aguacate (Persea americana), Bucare / Cachimbo (Erythrina poeppigiana), Cañafistol (Cassia moschata), Ceiba tolua (Pachira quinata), Eucalipto (Eucalyptus globulus), Guácimo (Guazuma ulmifolia), Guamo playero (Inga densiflora), Guanábano montañero/Tucuragua/Mapaso (Annona squamosa), Hobo (Spondias mombin), F. elastica, Matarratón (Gliricidia sepium), Melina (Gmelina arborea), Mora (Maclura tinctoria), Nauno (Pseudosamanea guachapelle / Albizzia guachapelle), Nigüito / Zurrumbo (Trema micrantha), Palma real (Attalea regia), Samán (Samanea saman), Sangro (Swartzia macrophylla), Tectona grandis y Yarumo (Cecropia peltata).

2) Cacao con sombrío $(\mathrm{Ca}+\mathrm{S})$, El cultivo de cacao en el área de estudio tiene como especies de sombrío las siguientes: E. poeppigiana, Candelero (Caraipa llanorum), S. mombin, Albizzia sp, G. arborea, Ocobo (Tabebuia rosea), S. saman, y C. peltata. Se considera que los lotes del cultivo de cacao con sombrío se deberán aumentar la cantidad de especies de sombrío teniendo en cuenta las condiciones climáticas locales y los requerimientos de la especie.

Las especies asociadas al cultivo del cacao en el área de estudio, que se identificaron en las parcelas temporales son: G. arborea, Cachimbo (Erythrina poepigiana), samán (S. saman), C. llanorum, Llovizno/ Yopo blanco (Albizzia sp).

3) Cítricos (C), Las especies que se identifican en las parcelas de C son: limón (Citrus limon), mandarina (Citrus reticulata) Naranja arrayana (Citrus sinensis), Tangelo (Citrus tangelo), la distancia de siembra recurrente en las áreas muestreadas son 7 x $7 \mathrm{~m}$ y 8 x $7 \mathrm{~m}$, densidades de siembra de 189 - 204 árboles/ha.

4) Sistema silvopastoril bajo (SSPB), Las especies identificadas en este sistema productivo son: abejón (Astronium graveolens), brasil / granadillo (Terminalia amazonica), caimán/mapurito (Fagara sp), $P$. quinata, flor amarillo (Tabebuia crysantha), G. ulmifolia, guayabo (Psidium guajava), S. mombin, laurel (Aniba perutilis), lechero (Sapium glandulosum), Mata palo (Ficus elastica), G. sepium, M. tinctoria, nauno (Pseudosamanea guachapelle), palma corozo (Aiphanes aculeata), teca (Tectona grandis), trompillo (Guarea trichiloides), tucuragua / Mapaso (Anona squamosa), turma de perro (Andira sp) y vara santa (Triplaris americana). Algunas de estas especies además de ser sombrío ocasionalmente se utilizan como especies para ramoneo
5) Sistema silvopastoril alto (SSPA), Las especies arbóreas y arbustivas identificadas para este tipo de uso del suelo en el corregimiento de Tacarimena son: A. graveolens, H. courbaril, Fagara sp, ciruelo (Prunus domestica), G. ulmifolia, Guarataro (Vitex orinoicensis), P. guajava, S. mombin, A. perutilis, F. elastica, G. sepium, M. tinctoria, $P$. guachapeIle/ Albizzia guachapelle, palma corozo (Aiphanes aculeata), pardillo (Cordia gerascanthus), trompillo (Guarea trichiliodes), T. americana y yopo (Anadenanthera peregrina/Albizzia peregrina).

6) Bosques de galería (BG) presenta las siguientes especies en estado fustal así: E. poeppigiana, C. Ilanorum, caracaro (Enterolobium cyclocarpum), C. pentandra, P. quinata, G. ulmifolia, I. densiflora, A. scuamosa, Guarataro (Vitex orinocensis), P. guajava, Higuerilla (Ricinus communis), S. mombin, Indio desnudo (Bursera simarouba), S. glandulosum, Mango (Manguifera indica), Mata palo (Ficus sp), Melina (Gmelina arborea), Mora (Maclura tinctoria), Onoto (Bixa orellana), A. regia, Paraparo/ Barbasco (Lonchocarpus utilis), S. saman, P. guachapelle, Sangro (Swartzia macrophylla/Swartzia parvoflora), T. grandis, G. trichiliodes, T, americana, C. peltata y A. peregrina.

7) Mata de monte (MM); las especies de flora son: A. graveolens, alcornoco (Bowdichia virgiliodes), H. courbaril, bototo (Cochospermum sp), Erythrina poeppigiana, cabo de hacha (Casearia sp), cabuyo (Couratari guianensis), Fagara sp, camoruco/ Camajón (Sterculia apetala), C. Ilanorum. E. ciclocarpum, C. moschata, ceiba bonga (Ceiba pentandra), P. quinata, chaparro (Curatella americana), T. crysantha, G. ulmifolia, gualanday (Jacaranda caucana), I. densiflora, A. scuamosa, S. glandulosum, $V$. orinocensis, higuerón (Ficus citrifolia), $S$. mombin, A. perutilis, Mata palo, M. ticntoria, $B$. orellana, A. regia, Palma Sarare (Syagrus sancona), L. utilis, punta de lanza (Vismia sp), quince días (Tapirira guianensis), S. macrophylla, G. trichiliodes, A. squamosa, C. corymbosa, T. america, C. peltata, A. peregrina y Albizzia sp.

El estudio contó con cinco repeticiones por cada uno de los sistemas estudiados.

Se establecieron parcelas temporales de muestreo de $1000 \mathrm{~m}^{2}$ cada una, en las cuales se midieron las variables dasométricas: altura total y diámetro del tronco a la altura del pecho (dap) para todos los árboles con dap $\geq 10 \mathrm{~cm}$. A los frutales y plantas de cacao se les midió el diámetro del tronco a una altura de $30 \mathrm{~cm}$ $\left(d_{30}\right)$ en parcelas de $400 \mathrm{~m}^{2}$. Las variables dasométri- 
cas se emplearon para estimar la biomasa individual arriba del suelo usando los modelos alométricos detallados en la Tabla 1. La información de la densidad de la madera se tomó del repositorio digital de Global Wood Density Data base (Zanne et al., 2009).

\section{Variación de las reservas de carbono total por cambios en el uso del suelo}

Se estimó el impacto de potenciales cambios de uso del suelo en la variación del $\mathrm{C}$ en la biomasa total, comparando el carbono almacenado en cada uno de los sistemas de uso del suelo estudiados, mediante matriz de doble entrada. Los valores se calcularon como la diferencia en el almacenamiento de carbono entre el uso futuro y el uso actual y eso por la constante estequiométrica (3.67), de modo de convertir los datos a $\mathrm{CO}_{2} \mathrm{e}$ (IPCC, 2003; IPCC, 2006). Los valores positivos constituyen incrementos de carbono y reflejan mitigación; mientras que los valores negativos son reducciones de $\mathrm{C}$ y en efecto emisión de $\mathrm{CO}_{2}$.

\section{Análisis estadístico}

El análisis estadístico consistió en un diseño experimental completamente aleatorio con siete sistemas de uso del suelo como tratamientos y cinco repeticiones. Se realizó un análisis de varianza previo a la comprobación de los supuestos y una prueba de comparación de medias con la prueba LSD Fisher $(\alpha=0,05)$. Los análisis se realizaron usando el software Infostat, versión estudiantil 2018.

\section{Resultados}

\section{Biomasa arriba y abajo del suelo}

La biomasa total, así como sus componentes arriba y abajo del suelo, presentaron diferencias estadísticas entre usos del suelo $(\mathrm{P}<0.05)$ (Figura 2). En promedio, la biomasa abajo del suelo representó el $17.6 \%$ del total. Las reservas de biomasa aérea en el área de estudio son importantes en todos los sistemas estudiados, con mayor énfasis en el BG y la MM con 132.5 y $77.3 \mathrm{Mg} / \mathrm{ha}$, respectivamente. En los SAF, se encontraron reservas de biomasa entre 27.7 y $53.0 \mathrm{Mg} / \mathrm{ha}$ (Figura 2). La biomasa abajo del suelo varió entre 6.4 y $25.3 \mathrm{Mg} / \mathrm{ha}$, siendo el $\mathrm{BG}$ y MM los de mayor contenido, mientras que el $\mathrm{Ca}+\mathrm{S}$ tenía el menor valor. La biomasa total varió entre 34.1 y 157.8 Mg/ha en los sistemas estudiados.

\section{Reservas de carbono en biomasa arriba del suelo}

El carbono almacenado en la biomasa total fue significativamente diferente entre sistemas de uso del suelo $(\mathrm{P}<0.05)$ (Figura 3). Los sistemas forestales presentaron la mayor captura de carbono en la biomasa total $(43.8$ y $74.2 \mathrm{Mg} /$ ha para MM y BG), los cuales superaron entre un 61 y $268 \%$ a los sistemas productivos (Figura 3). El carbono total almacenado en el sistema está entre 16.0 y $74.2 \mathrm{Mg} / \mathrm{ha}$, presentándose diferencias significativas $(\mathrm{P}<0.05)$ entre sistemas. El BG y la MM presentaron el mayor almacenamiento con 43.8 y $74.2 \mathrm{Mg} / \mathrm{ha}$, respectivamente; teniendo el primero de diferencias significativas $(\mathrm{P}<0,05)$ con $\mathrm{Ca}+\mathrm{S}$, SSPA, SSPB, SAF+ Plátano y $\mathrm{C}$ (16.0; 26.0; $28.6 ; 30.3$ y $36.6 \mathrm{Mg} / \mathrm{ha}$, respectivamente).

Tabla 1. Listado de Ecuaciones alométricas utilizadas en el estudio de reservas de carbono del Corregimiento de Tacarimena, Municipio de Yopal, Casanare, Colombia.

\begin{tabular}{|l|l|l|}
\hline \multicolumn{1}{|c|}{ Especie } & \multicolumn{1}{c|}{ Ecuación alométrica } & \multicolumn{1}{c|}{ Fuente } \\
\hline Frutales & $\mathrm{Ba}=10^{\wedge}\left(-1.11+2,64^{*} \log (\mathrm{dap})\right)$ & Andrade (2008) \\
\hline Acacia mangium & $\mathrm{Ba}=3.4+0,064^{*} \mathrm{dap}^{2}+1.0^{*} \mathrm{H}$ & Andrade et al., (1999) \\
\hline Theobroma cacao & $\mathrm{Ba}=10^{\wedge\left(-1.625+2.63^{*} \log (\mathrm{d} 30)\right)}$ & Andrade et al., (2008) \\
\hline Tectona grandis & $\mathrm{Ba}=10^{\wedge}\left(-0.815+2.382^{*} \log (\mathrm{dap})\right)$ & Pérez y Kanninen $(2003)$ \\
\hline Bosque húmedo tropical & $\mathrm{Ba}=\mathbf{e}^{\left(-2.289+0.937^{*} \operatorname{Ln}\left((\mathrm{dap})^{\wedge}(2)^{*}(\mathrm{H})^{*}(\mathrm{D})\right)\right.}$ & Álvarez et al., (2012) \\
\hline Gmelina arborea & $\mathrm{Ba}=\mathbf{e}^{\left(-3.38+\left(0.92^{*} \operatorname{Ln}\left(\left((\mathrm{dap})^{\wedge} 2\right)^{*}(\mathrm{H})\right)\right)\right.}$ & Onyekwelu $(2004)$ \\
\hline Palmas & $\mathrm{Ba}=6.66+12.826 \times \mathrm{H}^{0.5} \times \operatorname{Ln}(\mathrm{H})$ & WWF (2014) \\
\hline Biomasa abajo del suelo (raíces) & $\mathrm{Br}=\mathbf{e}^{\left(-1.0587+0.8836^{*} \operatorname{Ln}(\mathrm{B})\right)}$ & Cairns et al., (1997) \\
\hline
\end{tabular}

Notas: $\mathrm{R}^{2}$ ajustado: coeficiente de determinación ajustado; Ba: biomasa aérea (kg/individuo); dap: diámetro a la altura de pecho ( $\mathrm{m}$ ); $\mathrm{H}$ : altura total (m); log: logaritmo base 10; d30: diámetro del tronco a $30 \mathrm{~cm}$ de altura (cm); Ln: logaritmo natural (base e). D: densidad de la madera (g/ $\left.\mathrm{cm}^{3}\right)$. Br: Biomasa de raíces ( $\left.\mathrm{Mg} / \mathrm{ha}\right)$ B: Biomasa aérea total ( $\left.\mathrm{Mg} / \mathrm{ha}\right)$. 


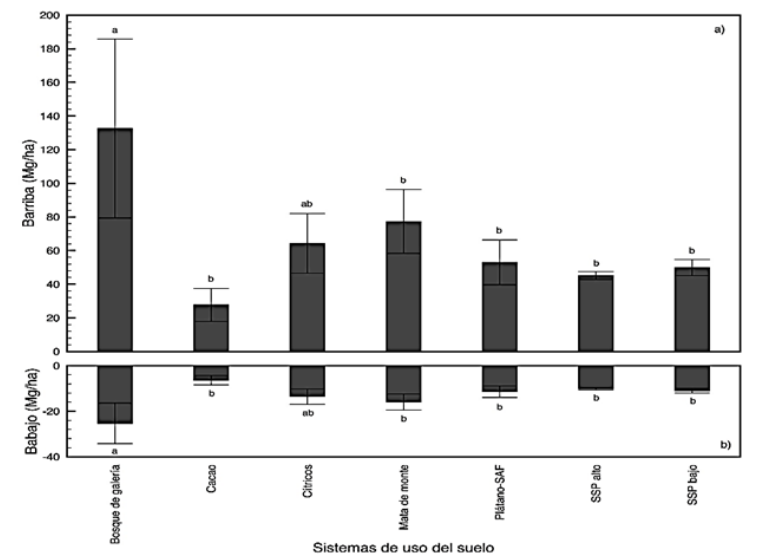

Figura 2. Biomasa arriba (B. arriba) (a) y abajo (B. abajo) (b) del suelo en sistemas de uso del suelo; Corregimiento de Tacarimena, Municipio de Yopal, Casanare, Colombia.: cacao con sombrío: Ca+S, SAF: sistemas agroforestales; SSP: sistema silvopastoril. Las barras de error corresponden al error estándar. Medias con letra común no son significativamente diferentes $(P>0.05)$.

Los resultados demuestran una alta variabilidad en el almacenamiento de carbono en la biomasa total.

\section{Impacto potencial en el almacenamiento de carbono por cambios en el uso del suelo}

La cantidad de carbono almacenado depende de la cobertura del suelo. De los usos en estudio, se encontró que el menor impacto en el almacenamiento de carbono se presenta en cacao con sombrío $(\mathrm{Ca}+\mathrm{S})$; si se cambia de este uso del suelo a sistema silvopastoril alto (SSPA), se capturan 36.6 Mg CO $2 /$ ha adicionales. En este mismo sentido, si se cambia de cacao $(\mathrm{Ca}+\mathrm{S})$ a $\mathrm{BG}$ se mitigan 213.4 $\mathrm{Mg} \mathrm{CO} 2 /$ ha, por el contrario cuando se da la pérdida de cobertura vegetal se ocasiona reducción en la captura de carbono con la consecuente emisión de $\mathrm{CO}_{2}$. Las mayores emisiones para este caso se generan si en el espacio donde hoy se tiene como uso del suelo BG, se estableciera $\mathrm{Ca}+\mathrm{S}$, con lo cual se emitiría un adicional de 213.4 Mg CO $2 /$ ha (Tabla 2). SSPB: Sistema silvopastoril bajo, reflejan emisiones de CO ${ }^{2}$ SSPA: Sistema silvopastoril alto; BG: Bosque de galería, MM: Mata de monte SAF+ plátano: $\mathrm{SAF}+$ plátano; $\mathrm{Ca}+\mathrm{S}$ : Cacao con sombrío, C: Cítricos. Los valores internos en la matriz corresponden a $\mathrm{Mg} \mathrm{CO} 2 \mathrm{e} / \mathrm{ha}$; los valores con fondo blanco representan ganancias de carbono (adicionalidad), mientras que los de fondo azul (negativos) reflejan emisiones de CO.

\section{Discusión}

Los resultados del presente estudio sobre biomasa guardan similitud con los presentados en estudios

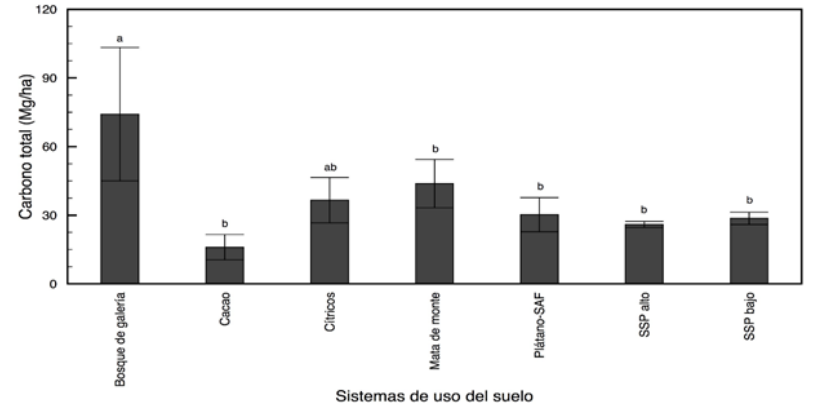

Figura 3. Carbono en biomasa total en los sistemas de uso del suelo en el corregimiento Tacarimena, Municipio de Yopal, Casanare, Colombia. SAF: sistemas agroforestales; SSP: sistema silvopastoril. Las barras de error corresponden al error estándar. Medias con letra común no son significativamente diferentes $(P>0.05)$.

de zonas climáticas similares como el de Marín et al., (2016). En este estudio se incluyeron especies maderables con frutales, tal como iguá (Albizia guachapelle), cedro (Cedrela odorata), nogal cafetero (Cordia alliodora), bayo (Acacia glomerata Benth), jobo (Spondias mombim), papayo (Carica papaya) y aguacate (Persea americana) con $122 \mathrm{Mg} /$ ha así como los SAF que solo albergan especies frutales y fijaron $72.5 \mathrm{Mg} / \mathrm{ha}$. La biomasa de los SAF en estudio es inferior a lo reportado en este estudio, lo cual se debe a la baja densidad del sombrío que presenta el $\mathrm{Ca}+\mathrm{S}$ y los $\mathrm{C}$ del área estudiada en donde se asocian especies como: Erythrina poeppigiana, Caraipa Ilanorum, Spondias mombin, Albizzia sp, Gmelina arborea, Tabebuia rosea, Samanea Saman y Cecropia peltata a baja densidad. En las parcelas de cítricos no se identifican especies forestales asociadas en los huertos.

Phillips et al., (2011, p. 29) "compara la biomasa calculada para bosques nativos por diferentes sistemas así: En la clasificación bioclimática de Holdridge, con una incertidumbre asociada a la estimación de 18,0\%... se observa que la biomasa aérea varía entre $91.4 \pm 11.1 \mathrm{Mg} / \mathrm{ha}$ y $334.5 \pm 126.8 \mathrm{Mg} / \mathrm{ha}$, mientras que la biomasa aérea promedio es de $255.2 \mathrm{Mg} / \mathrm{ha}$... en el sistema Land Cover Classification System... la biomasa aérea varía entre 119.4 $\pm 45.1 \mathrm{Mg} / \mathrm{ha}$ y $378.3 \pm 126.8 \mathrm{Mg} / \mathrm{ha}$, mientras que la biomasa aérea promedio es de $195.7 \mathrm{Mg} / \mathrm{ha}$ ". De otro lado, considerando el sistema de clasificación propuesto por Galindo et al., (2011), el IDEAM (2011) manifiesta que la biomasa aérea potencial en los bosques de Colombia varía entre 104.5 y $33.6 \mathrm{Mg} / \mathrm{ha}$, con un promedio de $294.0 \mathrm{Mg} / \mathrm{ha}$, los sistemas estudiados en Yopal presentaron valores inferiores a estos referentes. 
Tabla 2. Impacto potencial en el almacenamiento de carbono por cambios en el uso del suelo actual/ Uso futuro en Corregimiento Tacarimena, Municipio de Yopal, Casanare, Colombia.

\begin{tabular}{|c|c|c|c|c|c|c|c|c|}
\hline & \multicolumn{8}{|c|}{ Uso futuro } \\
\hline & Sistema & $\begin{array}{c}\text { Cacao con } \\
\text { sombrío } \\
(\mathrm{Ca}+\mathrm{S}) \\
(16.0 \mathrm{Mg} \\
\mathrm{C} / \mathrm{ha})\end{array}$ & $\begin{array}{c}\text { Sistema } \\
\text { silvopastoril } \\
\text { alto (SSPA) } \\
(26.0 \mathrm{Mg} \\
\mathrm{C} / \mathrm{ha})\end{array}$ & $\begin{array}{c}\text { silvopastoril } \\
\text { bajo SSPB } \\
\text { (28.6 Mg } \\
\text { C/ha) }\end{array}$ & $\begin{array}{c}\text { SAF+ } \\
\text { Plátano } \\
(30.3 \\
\mathrm{Mg} \\
\mathrm{C} / \mathrm{ha})\end{array}$ & $\begin{array}{c}\text { Cítricos } \\
\text { (C)(36.6 } \\
\text { Mg C/ } \\
\text { ha) }\end{array}$ & $\begin{array}{c}\text { Mata de } \\
\text { monte } \\
\text { MM (43.8 } \\
\text { Mg C/ha) }\end{array}$ & $\begin{array}{c}\text { Bosque de } \\
\text { galería BG } \\
\text { (74.2 Mg } \\
\mathrm{C} / \mathrm{ha})\end{array}$ \\
\hline \multirow{7}{*}{ 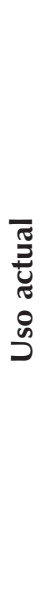 } & $\begin{array}{l}\text { Cacao con sombrío } \\
(\mathrm{Ca}+\mathrm{S})(16.0 \mathrm{Mg} \mathrm{C} / \mathrm{ha})\end{array}$ & 0 & 36.6 & 46.24 & 52.4 & 75.56 & 102.06 & 213.4 \\
\hline & $\begin{array}{l}\text { Sistema silvopastoril alto } \\
\text { (SSPA) (26.0 Mg C/ha) }\end{array}$ & -36.6 & 0 & 9.7 & 15.85 & 39.01 & 65.5 & 176.89 \\
\hline & $\begin{array}{l}\text { Sistema silvopastoril bajo } \\
\text { (SSPB) (28.6 Mg C/ha) }\end{array}$ & -46.24 & -9.7 & 0 & 6.17 & 29.32 & 55.82 & 167.2 \\
\hline & Plátano SAF (30.3 Mg C/ha) & -52.4 & -15.85 & -6.17 & 0 & 23.16 & 49.65 & 161.04 \\
\hline & Cítricos (C) (36.6 Mg C/ha) & -75.56 & -39.01 & -29.32 & -23.16 & 0 & 26.5 & 137.88 \\
\hline & $\begin{array}{l}\text { Mata de monte (MM) } \\
(43.8 \mathrm{Mg} \mathrm{C} / \mathrm{ha})\end{array}$ & -102.06 & -65.5 & -55.82 & -49.65 & -26.5 & 0 & 111.38 \\
\hline & $\begin{array}{l}\text { Bosque de galería (BG) } \\
\text { (74.2 Mg C/ha) }\end{array}$ & -213.4 & -176.89 & -167.2 & -161.04 & -137.88 & -111.38 & 0 \\
\hline
\end{tabular}

Fuente: Autores

La biomasa abajo del suelo estimada para la vegetación secundaria en este estudio (15.9-25.3 Mg/ha) presenta valores similares a los $20.4 \mathrm{Mg} / \mathrm{ha}$ estimados en ese mismo tipo de ecosistemas por Sierra et al., (2001) y superiores a los reportes de Fonseca et al., (2008) en bosques de 18 años.

El almacenamiento de carbono en los cacaotales de este estudio (16 C Mg/ha) resultaron similares a los hallazgos de Marín et al., (2016) en el Tolima, Colombia (19 Mg /ha) pero inferiores a los reportes de Ortiz et al., (2008) en cacaotales con Cordia alliodora en Changuinola, Panamá (43-62 Mg/ha). Estos resultados también son similares a los hallazgos de Arce et al., (2008) en fincas cacaoteras indígenas de Talamanca, Costa Rica: 36.5 C Mg/ha. Los resultados del presente estudio también resultan inferiores a los hallazgos de Patiño et al., (2018), quienes encontraron cerca de 85 $\mathrm{Mg} / \mathrm{ha}$ en biomasa total en SAF de cacao con sombrío de 10-15 años. Los estimados en Yopal resultaron inferiores debido a la inexistencia de árboles de sombrío.

En los SAF Ca+S y C se cuantificaron $16 \mathrm{C} \mathrm{Mg} / \mathrm{ha}$ y $36.6 \mathrm{C} \mathrm{Mg} / \mathrm{ha}$, respectivamente. Estos se cultivan bajo sombrío lo que ocasiona niveles medios de captura de carbono, tendría que indicarse que para aumentar la reserva de carbono en los sistemas productivos $\mathrm{Ca}+\mathrm{S}$, C debe aumentar y/o implementarse la instalación de especies de sombrío. De esta forma, se protege el cultivo de la incidencia de temperatura extrema y de paso se aumenta la captura carbono en los agrosistemas. En la región de San Martín (Perú), Concha et al., (2007) reportaron datos superiores a $40 \mathrm{C} \mathrm{Mg} / \mathrm{ha}$ para $\mathrm{SAF}$ con cacao. Estos sistemas incluían especies como el cedro (Cedrela odorata), shaina (Colubrina glandulosa) y frutales, como guamo (Inga sp), papaya (Carica papaya) y mango (Mangifera indica).

Los BG, que almacenaron el mayor carbono (74.2 Mg/ ha), presentaron valores bajos, los cuales pueden deberse al hecho que son bosques de galería que tienen limitaciones de los cauces y presión de la matriz agropecuaria, resultados similares reportaron Andrade et al., (2017). En la MM se cuantificó $43.8 \mathrm{Mg} /$ ha de carbono en biomasa total, lo cual es inferior a los valores presentados, lo cual podría deberse a la extracción de madera a la cual han venido siendo sometidas. La explicación a estas diferencias en los datos presentados en diversos estudios es atribuible a: 1) diferencias en la composición florística, 2) actividades de manejo y mantenimiento diferencial, 3) uso y aprovechamiento, 4) edad y 5) densidad del componente arbóreo.

La cantidad de carbono total almacenado en el BG concuerda con los datos de estudio de 16 tipos de bosques reportados que tienen carbono almacenado entre 16 y 
74.2 Mg/ha (Phillips et al., 2011). Por el contrario, el $\mathrm{Ca}+\mathrm{S}$ tienen las menores reservas de carbono, por tratarse de sistemas en los que el sombrío es disperso y escaso. Sin embargo, se califican como sumideros de carbono, siendo deseable aumentar el número de árboles de sombrío. Los resultados de ese estudio son mayores a los encontrados en Yopal, en donde solamente el sistema BG supera los $40 \mathrm{Mg} / \mathrm{ha}$, el valor reportado es $(66.3 \mathrm{Mg} / \mathrm{ha})$. Estas estimaciones y la literatura científica confirman la importancia de los SAF como estrategias para mitigar el cambio climático (Segura et al., 2008; Segura et al., 2012; Andrade et al., 2014).

El valor propuesto para almacenamiento de carbono en biomasa por el IPCC (2006) es de $141 \mathrm{Mg} /$ ha, el cual es superior al encontrado por el presente estudio en BG, lo cual puede causarse por las entresacas o talas selectivas de especies valiosas por su tamaño y calidad de la madera. Sin embargo, se consideran sumideros de carbono, además el BG y la MM no presentan actividades antrópicas por ubicarse en las rondas hídricas y en sitios donde los dueños de predio han tenido a bien conservar estas áreas cuya función es la regulación, la conservación de la diversidad biológica siendo áreas estratégicas de conservación.

Los sistemas silvopastoriles en la zona de estudio son usos del suelo con potencial para el almacenamiento de carbono. El mejoramiento de pasturas y el aumento de la cobertura arbórea con propósitos agroforestales pueden hacer que se presenten con potencial almacenamiento de carbono a nivel del área de estudio; además de tener bosques que permitan la protección de los recursos hídricos. El estado, por su parte, viene fomentando proyectos productivos a pequeña escala en el área de estudio empleando SAF con el fin de reducir la huella de carbono, tal como la producción de cacao y de café con sombrío y plátano asociado a cacao como sombrío en la etapa inicial.

El presente estudio encontró que el uso más deseado son los BG, cuya tala para pasar a Ca+ S, causaría emisiones de 213 Mg CO $2 /$ ha. Ordóñez et al., (2001) consideran como tareas prioritarias realizar estudios de emisión-captura de carbono en ecosistemas forestales que presenten procesos muy dinámicos de cambio de uso del suelo. Esto podría permitir, por un lado, estimar precisamente la densidad de carbono asociadas a los tipos de vegetación y, por el otro, desarrollar métodos de cuantificación y predicción de la emisión o captura de carbono bajo diferentes dinámicas de cambio de uso del suelo. De esta forma, se pueden ofrecer opciones de mitigación de GEl en el corto, mediano y largo plazo. Es decir, la captura de carbono permitirá contribuir directamente en la mitigación del fenómeno conocido como cambio climático.

En este mismo sentido, Andrade et al., (2016) han estudiado y documentado el impacto potencial por cambios en el uso del suelo, indicando que los mayores cambios en el almacenamiento de $\mathrm{C}$ se mantienen cuando se convierten áreas de pastura a bosques; por el contrario, al eliminar los bosques riparios y establecer arrozales el $\mathrm{C}$ tiende a disminuir generando emisiones de GEl en $12.3 \mathrm{Mg} \mathrm{CO} / \mathrm{ha}$. Lozano et al., (2011) afirman que actualmente uno de los principales problemas desde el punto de vista ambiental está referido a la pérdida de cobertura, y con ello a la fragmentación de los bosques, pérdida de la biodiversidad, alteración del recurso suelo, específicamente en cuanto a la erosión y la de generar $\mathrm{CO}_{2}$ a la atmósfera, en término de emisiones.

\section{Agradecimientos}

Los autores agradecen a los propietarios de predios del corregimiento de Tacarimena donde se tomó la información quienes desinteresadamente y de manera desprevenida permitieron el ingreso de personal a sus predios para tomar la información. De la misma forma, los autores agradecen al Grupo de Investigación PROECUT de la Universidad del Tolima por su apoyo en el análisis estadístico.

\section{Referencias}

Alcaldía de Yopal. 2013. Plan básico de ordenamiento territorial municipio de Yopal- Casanare, acuerdo 024/2013. Yopal, Colombia.

Alvarado J, Andrade-Castañeda HJ, Segura-Madrigal MA. Almacenamiento de carbono orgánico en suelos en sistemas de producción de café (coffea arábica l.) en el municipio del Líbano, Tolima, Colombia. Rev Colomb For, 2013;16(1): 31-21.

Álvarez E, Duque A, Saldarriaga J, Cabrera K, De las Salas G, Del Valle I, et al. Tree above-ground biomass allometries for carbon stocks estimation in the natural forests of Colombia. Forest Ecol Manag, 2012;267(1):297-308.

Andrade-Castañeda HJ. 1999. Dinámica productiva de sistemas silvopastoriles con Acacia mangium y Eucalyptus deglupta en el trópico húmedo. Tesis Mag. Sc. Turrialba, CR, CATIE. 70 p.

Andrade-Castañeda HJ, Segura-Madrigal MA. ¿Cómo construir modelos alométricos de volumen, biomasa o carbono de especies leñosas perennes?, Costa Rica. Agrofor Am, 2008;46(1):8996.

Andrade-Castañeda HJ, Segura-Madrigal MA, Rojas-Patiño AS. Carbono orgánico del suelo en bosques riparios, arrozales 
y pasturas en piedras, Tolima, Colombia. Agron Mesoam, 2015;27(2):233-241.

Andrade-Castañeda HJ, Segura-Madrigal MA, Canal-Daza DS, Huertas-Gonzales A, Mosos-Torres C. Composición florística y reservas de carbono en bosques ribereños en paisajes agropecuarios de la zona seca del Tolima, Colombia. Rev Biol Trop, 2017;65(4):1245-1260.

Arce N, Ortiz-Malavasi E, Villalobos M, Cordero S. Existencias de carbono en charrales y sistemas agroforestales de cacao y banano de fincas indígenas bribri y cabécar de Talamanca Costa Rica. Agrofor Am, 2008;46(1):30-33.

Burbano-Orjuela, $\mathrm{H}$. El carbono orgánico del suelo y su papel frente al cambio climático. Rev Cienc Agr, 2018;35(1):82-96.

Cairns MA, Brown S, Helmer EH, Baumgardner GA. Root biomass allocation in the word's upland forests. Revista Oecología, 1997; 111(1):1-11.

Concha J, Alegre JC, Pocomucha V. Determinación de las reservas de carbono en la biomasa aérea de sistemas agroforestales de Theobroma cacao L. en el Departamento de San Martín, Perú. Departamento Académico de Biología, Universidad Nacional Agraria La Molina, Lima - Perú. Ecol apl, 2007;6(1-2):75-82.

CORPORINOQUIA - Corporación Autónoma Regional de la Orinoquía. 2019. Actualización POMCA Plan de ordenación y manejo de la cuenca del Río Cravo Sur. Yopal, Colombia.

El Congreso de Colombia. 2018. Ley No. 1931 de 2018 por la cual se establecen directrices para la gestión del cambio climático. Gestor normativo de la función pública. Publicado en el Diario Oficial No. 50.667 de 27 de julio de 2018. Bogotá, Colombia

FAO - Organización de las Naciones Unidas para la Alimentación y la Agricultura. 2002. Captura de carbono en los suelos para un mejor manejo de la tierra. Basado en el trabajo de Michel Robert. Institut national de recherche agronomique. París, Francia.

Fonseca W, Alice F, Montero J, Toruño H, Leblanc H. Acumulación de biomasa y carbono en bosques secundarios y plantaciones forestales de (Vochysia guatemalensis e Hieronyma alchorneoides) en el Caribe de Costa Rica. 2008. Agrofor Am, 2008;46(1):57-64.

Galindo GA, Cabrera E, Vargas DM, Pabón HR, Cabrera KR, Yepes AP, Phillips JF, Navarrete DA, Duque AJ, García MC, Ordoñez MF. 2011. Estimación de la Biomasa Aérea usando Datos de Campo e información De Sensores Remotos. Instituto de Hidrología, Meteorología, y Estudios Ambientales. IDEAM. Bogotá D.C., Colombia. 52 p.

IGAC - Instituto Geográfico Agustín Codazzi. 2014. Estudio general de suelos y zonificación de tierras del Departamento de Casanare; escala 1:100.000. Bogotá, Colombia.

IPCC - Intergovernmental Panel on Climate Change. 2003. National Greenhouse Gas Inventories Programme Intergovernmental. Good Practice Guidance for Land Use Land-Use Change and Forestry. En IPCC Good Practice Guidance for LULUCF, Chapter 4: Supplementary Methods and Good Practice Guidance Arising from the Kyoto Protocol, Panel on Climate Change. pp. 113-116. Hayama, Kanagawa, Japón.
IPCC - Intergovernmental panel on climate change). 2006. Pautas para los inventarios nacionales de gases de efecto invernadero. Preparado por el Programa Nacional de Inventarios de Gases de Efecto Invernadero, Eggleston H.S., Buendia L., Miwa K., Ngara T. y Tanabe K. (eds). Publicado por: IGES, Japan.

IPCC - Intergovernmental Panel on Climate Change. 2013. Cambio climático: bases físicas. Contribución del grupo de trabajo I al quinto informe de evaluación del grupo intergubernamental de expertos sobre el cambio climático. IPCC, Ginebra, Suiza.

Lozano-Botache LA, Gómez-Aguiar FA, Valderrama-Chaves S. Estado de fragmentación de los bosques naturales en el norte del departamento del Tolima. Revista Tumbaga, 2011;6 (1):125140.

Marín-Q MDP, Andrade-Castañeda HJ, Sandoval AP. Fijación de carbono atmosférico en la biomasa total de sistemas de producción de cacao en el departamento del Tolima, Colombia. Rev UDCA Actual Divulg Cient, 2016; 19(2):351-360.

Mena VE, Andrade-Castañeda HJ, Navarro, CM. Biomasa y carbono almacenado en sistemas agroforestales con café y en bosques secundarios en gradientes altitudinales en Costa Rica. Rev Agrofor Neotrop, 2011;1(1):1-20.

MADS - Dirección de Cambio Climático. Estrategia Colombiana de Desarrollo Bajo en Carbono (ECDBC). 2011 CONPES 3700. Bogotá, Colombia.

Onyekwelu JC. Above-ground biomass production and biomass equations for even-aged Gmelina arborea (ROXB) plantations in south-western Nigeria. Biomass Bioenergy, 2004;26(1):3946.

Ordóñez, JAB; Masera O. Captura de carbono ante el cambio climático. Madera y Bosques, 2001;7(1):3-12. Bogotá, Colombia.

Ortiz A, Riascos L, Somarriba E. Almacenamiento y tasas de fijación de biomasa y carbono en sistemas agroforestales de cacao (Theobroma cacao) y laurel (Cordia alliodora) Avances de Investigación. Agrofor Am, 2008;46(1):26-29.

Patiño S, Suárez LN, Andrade-Castañeda HJ, Segura-Madrigal MA. Captura de carbono en biomasa en plantaciones forestales y sistemas agroforestales en Armero-Guayabal, Tolima, Colombia. Rev Investig Agrar Ambient, 2018;9(2):121-133.

Pérez-Cordero LD, Kanninen M. Above-Ground Biomass of Tectona grandis Plantations in Costa Rica. J Trop For Sci, 2003;15(1):199213.

Phillips JF, Duque AJ, Yepes AP, Cabrera KR, García MC, Navarrete DA, Álvarez E, Cárdenas D. 2011. Estimación de las reservas actuales (2010) de carbono almacenadas en la biomasa aérea en bosques naturales de Colombia. Estratificación, alometría y métodos analíticos. Instituto de Hidrología, Meteorología, y Estudios Ambientales IDEAM-. Bogotá D.C., Colombia. $68 \mathrm{p}$.

Segura-Madrigal MA, Andrade-Castañeda HJ. ¿Cómo construir modelos alométricos de volumen, biomasa o carbono de especies leñosas perennes? Costa Rica. Agrofor Am, 2008;46(1):90-100.

Segura-Madrigal MA, Andrade-Castañeda HJ. Huella de carbono en cadenas productivas de café (coffea arabica l.) con diferentes 
estándares de certificación en Costa Rica. Costa Rica. Rev Luna azul, 2012;35(1):60-77.

Sierra CA, del Valle Jl, Orrego SA. 2001. Ecuaciones de biomasa de raíces y sus tasas de acumulación en bosques sucesionales y maduros tropicales de Colombia. Simposio internacional Medición y monitoreo de la captura de carbono en ecosistemas forestales. Valdivia, Chile. 1-16.
WWF - Fondo Mundial para la Naturaleza. 2014. Estimación del carbono en la biomasa aérea de los bosques de la región de madre de Dios. Reporte. Lima, Perú.

Zanne AE, López G, Coomes DA, Llic J, Jansen S, Lewis SL, Miller RB, Swenson NG, Wiemann MC, Chave, J. 2009. Global Wood Density Database.xls.

Blanca Carvajal: https://orcid.org/0000-0002-3848-8959 Hernán Andrade: https://orcid.org/0000-0002-3398-294X 